\title{
Weighted Sobolev-Lieb-Thirring inequalities
}

Kazuya Tachizawa

\begin{abstract}
We give a weighted version of the Sobolev-Lieb-Thirring inequality for suborthonormal functions. In the proof of our result we use $\varphi$-transform of Frazier-Jawerth.
\end{abstract}

\section{Introduction}

In 1994 Edmunds and Ilyin proved a generalization of the Sobolev-LiebThirring inequality.

Theorem $1.1([2])$. Let $n \in \mathbb{N}, s>0$ and $p$ with

$$
\max \left(1, \frac{n}{2 s}\right)<p \leq 1+\frac{n}{2 s} \text {. }
$$

Then there exists a positive constant $c=c(p, n, s)$ such that for every family $\left\{\phi_{i}\right\}_{i=1}^{N}$ in $H^{s}\left(\mathbb{R}^{n}\right)$ which is orthonormal in $L^{2}\left(\mathbb{R}^{n}\right)$, we have

$$
\left\{\int_{\mathbb{R}^{n}} \rho(x)^{p /(p-1)} d x\right\}^{2 s(p-1) / n} \leq c \sum_{i=1}^{N}\left\|(-\Delta)^{s / 2} \phi_{i}\right\|^{2}
$$

where

$$
\rho(x)=\sum_{i=1}^{N}\left|\phi_{i}(x)\right|^{2}
$$

In this theorem $H^{s}\left(\mathbb{R}^{n}\right)$ denotes the Sobolev space of order $s$ and $\|\cdot\|$ is the norm of $L^{2}\left(\mathbb{R}^{n}\right)$. In [8] Lieb and Thirring proved this theorem for $s=1$ and applied it to the problem of the stability of matter. 
Ghidaglia, Marion and Temam proved (1.1) for $s \in \mathbb{N}$ under the suborthonormal condition on $\left\{\phi_{i}\right\}$, where $\left\{\phi_{i}\right\}_{i=1}^{N}$ in $L^{2}\left(\mathbb{R}^{n}\right)$ is called suborthonormal if the inequality

$$
\sum_{i, j=1}^{N} \xi_{i} \bar{\xi}_{j}\left(\phi_{i}, \phi_{j}\right) \leq \sum_{i=1}^{N}\left|\xi_{i}\right|^{2}
$$

holds for all $\xi_{i} \in \mathbb{C}, i=1, \ldots, N([4])$. They applied the inequality (1.1) to the estimate of the dimension of attractors associated with partial differential equations(c.f. [13]). In this paper we shall give a weighted version of (1.1) under suborthonormal condition on $\left\{\phi_{i}\right\}$. In the proof of our theorem we shall use Frazier-Jawerth's $\varphi$-transform ([3]).

For the statement of our result we need to recall the definition of $A_{p^{-}}$ weights (c.f. [5], [10]). By a cube in $\mathbb{R}^{n}$ we mean a cube which sides are parallel to coordinate axes. Let $w$ be a non-negative, locally integrable function on $\mathbb{R}^{n}$. We say that $w$ is an $A_{p}$-weight for $1<p<\infty$ if there exists a positive constant $C$ such that

$$
\frac{1}{|Q|} \int_{Q} w(x) d x\left(\frac{1}{|Q|} \int_{Q} w(x)^{-1 /(p-1)} d x\right)^{p-1} \leq C
$$

for all cubes $Q \subset \mathbb{R}^{n}$. The infimum of the constant $C$ is called the $A_{p}$-constant of $w$. For example, $w(x)=|x|^{\alpha}$ is an $A_{p}$-weight when $-n<\alpha<n(p-1)$.

We say that $w$ is an $A_{1}$-weight if there exists a positive constant $C$ such that

$$
\frac{1}{|Q|} \int_{Q} w(y) d y \leq C w(x) \quad \text { a.e. } x \in Q
$$

for all cubes $Q \subset \mathbb{R}^{n}$. The infimum of the constant $C$ is called the $A_{1}$-constant of $w$. Let $A_{p}$ be the class of $A_{p}$-weights. The inclusion $A_{p} \subset A_{q}$ holds for $p<q$.

For a nonnegative, locally integrable function $w$ on $\mathbb{R}^{n}$ we define

$$
L^{p}(w)=\left\{f: \text { measurable on } \mathbb{R}^{n}, \int_{\mathbb{R}^{n}}|f(x)|^{p} w(x) d x<\infty\right\} .
$$

For $\nu \in \mathbb{Z}$ and $k \in \mathbb{Z}^{n}$ the cube $Q$ defined by

$$
Q=Q_{\nu k}=\left\{\left(x_{1}, \ldots, x_{n}\right): k_{i} \leq 2^{\nu} x_{i}<k_{i}+1, i=1, \ldots, n\right\}
$$

is called a dyadic cube in $\mathbb{R}^{n}$. Let $\mathcal{Q}$ be the set of all dyadic cubes in $\mathbb{R}^{n}$. For any $Q \in \mathcal{Q}$ there exists a unique $Q^{\prime} \in \mathcal{Q}$ such that $Q \subset Q^{\prime}$ and the side-length of $Q^{\prime}$ is double of that of $Q$. We call $Q^{\prime}$ the parent of $Q$.

For $s>0$ and $f \in C_{0}^{\infty}\left(\mathbb{R}^{n}\right)$ we define via inverse Fourier transform

$$
(-\Delta)^{s / 2} f(x)=\mathcal{F}^{-1}\left(|\xi|^{s} \hat{f}\right)(x) \text {. }
$$


Let $w \in A_{2}$ and $\mathcal{H}^{s}(w)$ be the completion of $C_{0}^{\infty}\left(\mathbb{R}^{n}\right)$ with respect to the norm

$$
\|f\|_{\mathcal{H}^{s}(w)}=\left\{\int_{\mathbb{R}^{n}}\left|(-\Delta)^{s / 2} f(x)\right|^{2} w(x) d x+\|f\|^{2}\right\}^{1 / 2} .
$$

We remark that for $f \in C_{0}^{\infty}\left(\mathbb{R}^{n}\right)$ we have

$$
\int_{\mathbb{R}^{n}}\left|(-\Delta)^{s / 2} f(x)\right|^{2} w(x) d x<\infty
$$

because

$$
\left|(-\Delta)^{s / 2} f(x)\right| \leq \frac{c}{(1+|x|)^{n}} \quad\left(x \in \mathbb{R}^{n}\right)
$$

and

$$
\int_{\mathbb{R}^{n}} \frac{w(x)}{(1+|x|)^{2 n}} d x<\infty
$$

(c.f. [10, p. 209]).

Let $f \in \mathcal{H}^{s}(w)$ and $\left\{f_{i}\right\}_{i=1}^{\infty}$ be a sequence in $C_{0}^{\infty}\left(\mathbb{R}^{n}\right)$ such that

$$
\left\|f-f_{i}\right\|_{\mathcal{H}^{s}(w)} \rightarrow 0(i \rightarrow \infty) .
$$

This means that there exist $g_{1} \in L^{2}\left(\mathbb{R}^{n}\right)$ and $g_{2} \in L^{2}(w)$ such that

$$
\left\|g_{1}-f_{i}\right\| \rightarrow 0 \quad \text { and } \quad \int_{\mathbb{R}^{n}}\left|g_{2}(x)-(-\Delta)^{s / 2} f_{i}(x)\right|^{2} w(x) d x \rightarrow 0
$$

as $i \rightarrow \infty$. We denote $(-\Delta)^{s / 2} f=g_{2}$. We remark that $g_{1} \equiv 0$ means $g_{2} \equiv 0$. In fact, for any $\varphi \in C_{0}^{\infty}\left(\mathbb{R}^{n}\right)$, we have

$$
\int_{\mathbb{R}^{n}} g_{2} \bar{\varphi} d x=\lim _{i \rightarrow \infty} \int_{\mathbb{R}^{n}}(-\Delta)^{s / 2} f_{i} \bar{\varphi} d x=\lim _{i \rightarrow \infty} \int_{\mathbb{R}^{n}} f_{i} \overline{(-\Delta)^{s / 2} \varphi} d x=0 .
$$

Hence we have $g_{2} \equiv 0$. This means that we can identify $\mathcal{H}^{s}(w)$ as a subspace of $L^{2}\left(\mathbb{R}^{n}\right)$.

The following is the main result of this paper.

Theorem 1.2. Let $n \in \mathbb{N}, s>0$, and

$$
\max \left(1, \frac{n}{2 s}\right)<p \leq 1+\frac{n}{2 s} .
$$

Let $w \in A_{2}$. If $2 s<n$, then we assume that $w^{-n /(2 s)} \in A_{n /(2 s)}$. If $2 s \geq n$, then we assume that $w^{-n /(2 s)} \in A_{p}$ and

$$
\int_{Q^{\prime}} w d x \leq 2^{2 s} \int_{Q} w d x
$$

for all dyadic cubes $Q \in \mathcal{Q}$ and its parent $Q^{\prime}$. 
Then there exists a positive constant c such that for every family $\left\{\phi_{i}\right\}_{i=1}^{N}$ in $\mathcal{H}^{s}(w)$ which is suborthonormal in $L^{2}\left(\mathbb{R}^{n}\right)$, we have

$\left\{\int_{\mathbb{R}^{n}} \rho(x)^{p /(p-1)} w(x)^{n /(2 s(p-1))} d x\right\}^{2 s(p-1) / n} \leq c \sum_{i=1}^{N} \int_{\mathbb{R}^{n}}\left|(-\Delta)^{s / 2} \phi_{i}(x)\right|^{2} w(x) d x$,

where

$$
\rho(x)=\sum_{i=1}^{N}\left|\phi_{i}(x)\right|^{2}
$$

and $c$ depends only on $n, s, p, A_{2}$-constant of $w$, and $A_{n /(2 s)}$ or $A_{p}$-constant of $w^{-n /(2 s)}$.

When $2 s<n$, an example of weight function $w$ is given by $w(x)=|x|^{\alpha}$ for $-n+2 s<\alpha<2 s$. When $2 s>n$, an example of weight function $w$ is given by $w(x)=|x|^{\alpha}$ for $0 \leq \alpha<\min \{2 s-n, n\}$ (c.f. [12, Section 4]). When $2 s=n$, the condition (1.2) means $w$ is equivalent to a constant almost everywhere(c.f.[12, Proposition 4.1]).

\section{Preliminaries}

Let $\psi$ be a function which satisfies the following conditions.

(A1) $\psi \in \mathcal{S}\left(\mathbb{R}^{n}\right)$.

(A2) $\operatorname{supp} \hat{\psi} \subset\left\{\xi \in \mathbb{R}^{n}: \frac{1}{2} \leq|\xi| \leq 2\right\}$

(A3) $|\hat{\psi}(\xi)| \geq c>0$ if $\frac{3}{5} \leq|\xi| \leq \frac{5}{3}$.

(A4) $\sum_{\nu \in \mathbb{Z}}\left|\hat{\psi}\left(2^{\nu} \xi\right)\right|^{2}=1$ for all $\xi \neq 0$.

For $\nu \in \mathbb{Z}, k \in \mathbb{Z}^{n}$ and $Q=Q_{\nu k}$, we set

$$
\psi_{Q}(x)=2^{\nu n / 2} \psi\left(2^{\nu} x-k\right) \quad\left(x \in \mathbb{R}^{n}\right) .
$$

Let $M$ be the Hardy-Littlewood maximal operator, that is,

$$
M(f)(x)=\sup _{x \in Q} \frac{1}{|Q|} \int_{Q}|f(y)| d y,
$$

where $f$ is a locally integrable function on $\mathbb{R}^{n}$ and the supremum is taken over all cubes $Q$ which contain $x$. 
Proposition 2.1. (i) Let $1<p<\infty$ and $w$ be a non-negative locally integrable function on $\mathbb{R}^{n}$. Then there exists a positive constant c such that

$$
\int_{\mathbb{R}^{n}} M(f)^{p} w d x \leq c \int_{\mathbb{R}^{n}}|f|^{p} w d x
$$

for all $f \in L^{p}(w)$ if and only if $w \in A_{p}$. The constant $c$ depends only on $n, p$ and $A_{p}$-constant of $w$.

(ii) Let $1<p<\infty$ and $w \in A_{p}$. Then there exists a $q \in(1, p)$ such that $w \in A_{q}$.

(iii) Let $0<\tau<1$ and $f$ be a locally integrable function on $\mathbb{R}^{n}$ such that $M(f)(x)<\infty$ a.e.. Then $(M(f))^{\tau} \in A_{1}$ and the $A_{1}$-constant of $(M(f))^{\tau}$ depends only on $n$ and $\tau$.

(iv) Let $1 \leq p<\infty$ and $w \in A_{p}$. Then there exists a positive constant $c$ such that

$$
\int_{2 Q} w d x \leq c \int_{Q} w d x
$$

for all cubes $Q \in \mathbb{R}^{n}$, where $2 Q$ denotes the double of $Q$ and c depend only on $n$ and $A_{p}$-constant of $w$.

The proofs of these facts are in [5, Chapter IV] or [10, Chapter V].

\section{Proof of Theorem 1.2}

The suborthonormal condition on $\left\{\phi_{i}\right\}$ is equivalent to the inequality

$$
\sum_{i=1}^{N}\left|\left(\phi_{i}, f\right)\right|^{2} \leq\|f\|^{2}
$$

for all $f \in L^{2}\left(\mathbb{R}^{n}\right)$ (c.f. $\left.[1, \mathrm{p} 57]\right)$. We shall prove the inequality

$$
\begin{aligned}
\left\{\int_{\mathbb{R}^{n}} \rho(x)^{p /(p-1)} w\right. & \left.(x)^{n /(2 s(p-1))} d x\right\}^{2 s(p-1) / n} \\
& \leq c K^{2 s p / n-1} \sum_{i=1}^{N} \int_{\mathbb{R}^{n}}\left|(-\Delta)^{s / 2} \phi_{i}(x)\right|^{2} w(x) d x
\end{aligned}
$$

under the assumption

$$
\sum_{i=1}^{N}\left|\left(\phi_{i}, f\right)\right|^{2} \leq K\|f\|^{2}
$$

for all $f \in L^{2}\left(\mathbb{R}^{n}\right)$ where $K$ is a positive constant. 
This is equivalent to the statement of Theorem 1.2. We remark that $K$ may depend on $\left\{\phi_{i}\right\}$. For example, the inequality (3.1) says that

$$
\left\{\int_{\mathbb{R}^{n}}|\phi|^{2 p /(p-1)} w^{n /(2 s(p-1))} d x\right\}^{2 s(p-1) / n} \leq c\|\phi\|^{4 s p / n-2} \int_{\mathbb{R}^{n}}\left|(-\Delta)^{s / 2} \phi\right|^{2} w d x
$$

holds for all $\phi \in \mathcal{H}^{s}(w)$ under suitable condition on $s, p, n$ and $w$ because

$$
|(\phi, f)|^{2} \leq\|\phi\|^{2}\|f\|^{2}
$$

for all $f \in L^{2}\left(\mathbb{R}^{n}\right)$.

First we assume $\phi_{i} \in C_{0}^{\infty}\left(\mathbb{R}^{n}\right), i=1, \ldots, N$. Let

$$
V(x)=\delta_{1} \rho(x)^{1 /(p-1)} w(x)^{n /(2 s(p-1))}
$$

where the value of the constant $\delta_{1}>0$ will be given later. Since

$$
\rho(x)=\sum_{i=1}^{N}\left|\phi_{i}(x)\right|^{2}
$$

is a bounded function with compact support and $w^{n /(2 s(p-1))}$ is locally integrable by the assumption $w^{-n /(2 s)} \in A_{p}$, we have

$$
\int_{\mathbb{R}^{n}} V^{p} w^{-n /(2 s)} d x<\infty
$$

We may also assume that

$$
0<\int_{\mathbb{R}^{n}} V^{p} w^{-n /(2 s)} d x
$$

By (ii) of Proposition 2.1 there exists a constant $\kappa$ such that

$$
1<\kappa<p \quad \text { and } \quad w^{-n /(2 s)} \in A_{p / \kappa} .
$$

We set

$$
v(x)=M\left(V^{\kappa}\right)(x)^{1 / \kappa} .
$$

Then (i) of Proposition 2.1 leads to

$$
\int_{\mathbb{R}^{n}} v^{p} w^{-n /(2 s)} d x=\int_{\mathbb{R}^{n}} M\left(V^{\kappa}\right)^{p / \kappa} w^{-n /(2 s)} d x \leq c_{1} \int_{\mathbb{R}^{n}} V^{p} w^{-n /(2 s)} d x<\infty
$$

Furthermore $v$ is an $A_{1}$-weight by (iii) of Proposition 2.1 . 
We have the following lemmas.

Lemma 3.1. For $s>0$ and $w \in A_{2}$ there exists a positive constant $\alpha$ such that

$$
\alpha \sum_{Q \in \mathcal{Q}}|Q|^{-2 s / n}\left|\left(f, \psi_{Q}\right)\right|^{2} \frac{1}{|Q|} \int_{Q} w d x \leq \int_{\mathbb{R}^{n}}\left|(-\Delta)^{s / 2} f\right|^{2} w d x
$$

for all $f \in C_{0}^{\infty}\left(\mathbb{R}^{n}\right)$, where $\alpha$ is given by

$$
\alpha^{-1}=c \max _{|\sigma| \leq n}\left\|\partial^{\sigma} \hat{\psi}\right\|_{\infty}^{2}
$$

and $c$ is a constant depending only on $n, s$ and $A_{2}$-constant of $w$.

Lemma 3.2. For $v \in A_{2}$ there exist positive constants $\beta$ and $\beta^{\prime}$ such that

$$
\beta^{\prime} \sum_{Q \in \mathcal{Q}}\left|\left(f, \psi_{Q}\right)\right|^{2} \frac{1}{|Q|} \int_{Q} v d x \leq \int_{\mathbb{R}^{n}}|f|^{2} v d x \leq \beta \sum_{Q \in \mathcal{Q}}\left|\left(f, \psi_{Q}\right)\right|^{2} \frac{1}{|Q|} \int_{Q} v d x
$$

for all $f \in C_{0}^{\infty}\left(\mathbb{R}^{n}\right)$, where $\beta$ is given by

$$
\beta=c \max _{|\sigma| \leq n}\left\|\partial^{\sigma} \hat{\psi}\right\|_{\infty}^{2}
$$

and $c$ is a constant depending only on $n$ and $A_{2}$-constant of $v$.

The proof of Lemmas 3.1 and 3.2 are in [11, Prop. 2.2 and Lemma 3.2]. We shall give the proof in Section 5 for the reader's convenience because the dependence of $\psi$ in $\alpha$ and $\beta$ is not explained in [11].

For $f \in C_{0}^{\infty}\left(\mathbb{R}^{n}\right)$ we have

$$
\int_{\mathbb{R}^{n}}|f|^{2} V d x \leq \int_{\mathbb{R}^{n}}|f|^{2} v d x \leq \beta \sum_{Q \in \mathcal{Q}}\left|\left(f, \psi_{Q}\right)\right|^{2} \frac{1}{|Q|} \int_{Q} v d x
$$

where we used Lemma 3.2. Hence by Lemma 3.1

$$
\begin{aligned}
& \int_{\mathbb{R}^{n}}\left|(-\Delta)^{s / 2} f\right|^{2} w d x-\int_{\mathbb{R}^{n}} V|f|^{2} d x \\
& \quad \geq \alpha \sum_{Q \in \mathcal{Q}}|Q|^{-2 s / n}\left|\left(f, \psi_{Q}\right)\right|^{2} \frac{1}{|Q|} \int_{Q} w d x-\beta \sum_{Q \in \mathcal{Q}}\left|\left(f, \psi_{Q}\right)\right|^{2} \frac{1}{|Q|} \int_{Q} v d x .
\end{aligned}
$$

Now we set

$$
\mathcal{I}=\left\{Q \in \mathcal{Q}: \beta \int_{Q} v d x>\alpha|Q|^{-2 s / n} \int_{Q} w d x\right\} .
$$

Let $\left\{\mu_{k}\right\}_{1 \leq k}$ be the non-decreasing rearrangement of

$$
\left\{\alpha|Q|^{-2 s / n-1} \int_{Q} w d x-\beta|Q|^{-1} \int_{Q} v d x\right\}_{Q \in \mathcal{I}} .
$$

We will show that this rearrangement is possible in the proof of Lemma 3.3. 
74 K. TACHIZAWA

When

$$
\mu_{k}=\alpha|Q|^{-2 s / n-1} \int_{Q} w d x-\beta|Q|^{-1} \int_{Q} v d x
$$

we define $\Psi_{k}=\psi_{Q}$. Then we have by (3.5)

$$
\begin{aligned}
& \sum_{i=1}^{N} \int_{\mathbb{R}^{n}}\left|(-\Delta)^{s / 2} \phi_{i}\right|^{2} w d x-\sum_{i=1}^{N} \int_{\mathbb{R}^{n}} V\left|\phi_{i}\right|^{2} d x \\
& \quad \geq \sum_{i=1}^{N} \sum_{Q \in \mathcal{Q}}\left|\left(\phi_{i}, \psi_{Q}\right)\right|^{2}\left\{\alpha|Q|^{-2 s / n-1} \int_{Q} w d x-\beta|Q|^{-1} \int_{Q} v d x\right\} \\
& \quad \geq \sum_{i=1}^{N} \sum_{k} \mu_{k}\left|\left(\phi_{i}, \Psi_{k}\right)\right|^{2}=\sum_{k} \mu_{k} \sum_{i=1}^{N}\left|\left(\phi_{i}, \Psi_{k}\right)\right|^{2} \\
& \quad \geq-K\|\psi\|^{2} \sum_{k}\left|\mu_{k}\right| \geq-K\|\psi\|^{2}\left(\sum_{k}\left|\mu_{k}\right|^{\gamma}\right)^{1 / \gamma}
\end{aligned}
$$

where $\gamma=p-n /(2 s) \in(0,1]$ and we used (3.2).

Now the following lemma holds.

\section{Lemma 3.3.}

$$
\sum_{k}\left|\mu_{k}\right|^{\gamma} \leq c \int_{\mathbb{R}^{n}} v^{p} w^{-n /(2 s)} d x
$$

where $c$ is given by

$$
c=c^{\prime} \max _{|\sigma| \leq n}\left\|\partial^{\sigma} \hat{\psi}\right\|_{\infty}^{n / s+2 p}
$$

and $c^{\prime}$ depends only on $n, s, p$ and $w$.

The proof of this lemma will be given in Section 4. By Lemma 3.3 and (3.4) the last quantity in (3.8) is estimated from below by

$$
\begin{gathered}
-c K\left(\int_{\mathbb{R}^{n}} V^{p} w^{-n /(2 s)} d x\right)^{1 / \gamma} \\
\quad=-c K \delta_{1}^{p /(p-n /(2 s))}\left(\int_{\mathbb{R}^{n}} \rho^{p /(p-1)} w^{n /(2 s(p-1))} d x\right)^{1 /(p-n /(2 s))},
\end{gathered}
$$

where

$$
c=c^{\prime}\|\psi\|^{2} \max _{|\sigma| \leq n}\left\|\partial^{\sigma} \hat{\psi}\right\|_{\infty}^{(4 p s+2 n) /(2 p s-n)}
$$

and $c^{\prime}$ depends only on $n, s, p$ and $w$. We may take the infimum of the above constant with respect to possible $\psi$ and replace $c$ by this infimum. 
Let

$$
\delta_{1}=\delta_{2} K^{1-2 s p / n}\left(\int_{\mathbb{R}^{n}} \rho^{p /(p-1)} w^{n /(2 s(p-1))} d x\right)^{2 s(p-n /(2 s)-1) / n},
$$

where $\delta_{2}$ is a positive constant. Then we have by (3.7)

$$
\begin{aligned}
\sum_{i=1}^{N} \int_{\mathbb{R}^{n}}\left|(-\Delta)^{s / 2} \phi_{i}\right|^{2} w d x \\
\geq \delta_{2} K^{1-2 s p / n}\left(\int_{\mathbb{R}^{n}} \rho^{p /(p-1)} w^{n /(2 s(p-1))} d x\right)^{2 s(p-1) / n} \\
\quad-c K \delta_{2}^{p /(p-n /(2 s))} K^{-2 s p / n}\left(\int_{\mathbb{R}^{n}} \rho^{p /(p-1)} w^{n /(2 s(p-1))} d x\right)^{2 s(p-1) / n} \\
=\left\{\delta_{2}-c \delta_{2}^{p /(p-n /(2 s))}\right\} K^{1-2 s p / n}\left(\int_{\mathbb{R}^{n}} \rho^{p /(p-1)} w^{n /(2 s(p-1))} d x\right)^{2 s(p-1) / n} .
\end{aligned}
$$

If we take $\delta_{2}$ small enough, then we get the inequality (3.1) because $1<p /(p-n /(2 s))$.

Next we shall show (3.1) for $\phi_{i} \in \mathcal{H}^{s}(w), i=1, \ldots, N$. First we show

$$
\mathcal{H}^{s}(w) \subset L^{2 p /(p-1)}\left(w^{n /(2 s(p-1))}\right) .
$$

Let $h \in \mathcal{H}^{s}(w)$. Then there exists a sequence $\left\{h_{m}\right\}_{m=1}^{\infty} \subset C_{0}^{\infty}\left(\mathbb{R}^{n}\right)$ such that $\left\|h-h_{m}\right\|_{\mathcal{H}^{s}(w)} \rightarrow 0(m \rightarrow \infty)$. Since we proved that (3.3) holds for $h_{m} \in$ $C_{0}^{\infty}\left(\mathbb{R}^{n}\right)$, we get

$$
\left\{\int_{\mathbb{R}^{n}}\left|h_{m}\right|^{2 p /(p-1)} w^{n /(2 s(p-1))} d x\right\}^{2 s(p-1) / n} \leq c\left\|h_{m}\right\|^{4 s p / n-2} \int_{\mathbb{R}^{n}}\left|(-\Delta)^{s / 2} h_{m}\right|^{2} w d x,
$$

where $c$ does not depend on $h_{m}$. Since $4 s p / n-2>0$ and $\left\{h_{m}\right\}$ is a Cauchy sequence in $\mathcal{H}^{s}(w)$, the above inequality says that $\left\{h_{m}\right\}$ is a Cauchy sequence in $L^{2 p /(p-1)}\left(w^{n /(2 s(p-1))}\right)$. Let $g$ be the limit of $\left\{h_{m}\right\}$ in $L^{2 p /(p-1)}\left(w^{n /(2 s(p-1))}\right)$. For any compact set $E$ in $\mathbb{R}^{n}$ we have

$$
\begin{aligned}
\int_{E}\left|g-h_{m}\right| d x \leq & \left(\int_{E}\left|g-h_{m}\right|^{2 p /(p-1)} w^{n /(2 s(p-1))} d x\right)^{(p-1) /(2 p)} \\
& \times\left(\int_{E} w^{-n /(2 s(p+1))} d x\right)^{(p+1) /(2 p)}
\end{aligned}
$$

Since $w^{-n /(2 s)}$ is locally integrable by the assumption $w^{-n /(2 s)} \in A_{n /(2 s)}$ or $w^{-n /(2 s)} \in A_{p}$, we get $h_{m} \rightarrow g$ in $L_{l o c}^{1}\left(\mathbb{R}^{n}\right)$ as $m \rightarrow \infty$. Hence we have $g=h$ and (3.9). 
Furthermore we have

$$
\left\{\int_{\mathbb{R}^{n}}|h|^{2 p /(p-1)} w^{n /(2 s(p-1))} d x\right\}^{2 s(p-1) / n} \leq c\|h\|^{4 s p / n-2} \int_{\mathbb{R}^{n}}\left|(-\Delta)^{s / 2} h\right|^{2} w d x
$$

We fix a positive number $\varepsilon$. Let $\chi_{1}, \ldots, \chi_{N}$ be functions in $C_{0}^{\infty}\left(\mathbb{R}^{n}\right)$ such that

$$
\sum_{i=1}^{N}\left\|\phi_{i}-\chi_{i}\right\|_{\mathcal{H}^{s}(w)}^{2}<\varepsilon
$$

Now the inequalities

$$
\begin{aligned}
\sum_{i=1}^{N}\left|\left(\chi_{i}, f\right)\right|^{2} & \leq 2 \sum_{i=1}^{N}\left|\left(\chi_{i}-\phi_{i}, f\right)\right|^{2}+2 \sum_{i=1}^{N}\left|\left(\phi_{i}, f\right)\right|^{2} \\
& \leq 2 \sum_{i=1}^{N}\left\|\chi_{i}-\phi_{i}\right\|^{2}\|f\|^{2}+2 K\|f\|^{2} \leq 2(K+\varepsilon)\|f\|^{2}
\end{aligned}
$$

hold for all $f \in L^{2}\left(\mathbb{R}^{n}\right)$. On the other hand

$$
\begin{aligned}
\left\{\int_{\mathbb{R}^{n}}\right. & \left.\left(\sum_{i=1}^{N}\left|\phi_{i}-\chi_{i}\right|^{2}\right)^{p /(p-1)} w^{n /(2 s(p-1))} d x\right\}^{2 s(p-1) / n} \\
& \leq\left\{\sum_{i=1}^{N}\left(\int_{\mathbb{R}^{n}}\left|\phi_{i}-\chi_{i}\right|^{2 p /(p-1)} w^{n /(2 s(p-1))} d x\right)^{(p-1) / p}\right\}^{2 s p / n} \\
& \leq N^{2 s p / n-1} \sum_{i=1}^{N}\left(\int_{\mathbb{R}^{n}}\left|\phi_{i}-\chi_{i}\right|^{2 p /(p-1)} w^{n /(2 s(p-1))} d x\right)^{2 s(p-1) / n} \\
& \leq c N^{2 s p / n-1} \sum_{i=1}^{N}\left\|\phi_{i}-\chi_{i}\right\|^{4 s p / n-2} \int_{\mathbb{R}^{n}}\left|(-\Delta)^{s / 2} \phi_{i}-(-\Delta)^{s / 2} \chi_{i}\right|^{2} w d x \\
& \leq c N^{2 s p / n-1} \varepsilon^{2 s p / n-1} \sum_{i=1}^{N} \int_{\mathbb{R}^{n}}\left|(-\Delta)^{s / 2} \phi_{i}-(-\Delta)^{s / 2} \chi_{i}\right|^{2} w d x \\
& \leq c N^{2 s p / n-1} \varepsilon^{2 s p / n},
\end{aligned}
$$

where we used (3.10). Therefore

$$
\begin{gathered}
\left\{\int_{\mathbb{R}^{n}}\left(\sum_{i=1}^{N}\left|\phi_{i}\right|^{2}\right)^{p /(p-1)} w^{n /(2 s(p-1))} d x\right\}^{2 s(p-1) / n} \\
\leq\left\{\int_{\mathbb{R}^{n}}\left(2 \sum_{i=1}^{N}\left|\phi_{i}-\chi_{i}\right|^{2}+2 \sum_{i=1}^{N}\left|\chi_{i}\right|^{2}\right)^{p /(p-1)} w^{n /(2 s(p-1))} d x\right\}^{2 s(p-1) / n}
\end{gathered}
$$




$$
\begin{aligned}
& \leq 2^{2 s p / n}\left[\left\{\int_{\mathbb{R}^{n}}\left(\sum_{i=1}^{N}\left|\phi_{i}-\chi_{i}\right|^{2}\right)^{p /(p-1)} w^{n /(2 s(p-1))} d x\right\}^{(p-1) / p}\right. \\
& \left.+\left\{\int_{\mathbb{R}^{n}}\left(\sum_{i=1}^{N}\left|\chi_{i}\right|^{2}\right)^{p /(p-1)} w^{n /(2 s(p-1))} d x\right\}^{(p-1) / p}\right]^{2 s p / n} \\
& \leq 2^{4 s p / n-1}\left\{\int_{\mathbb{R}^{n}}\left(\sum_{i=1}^{N}\left|\phi_{i}-\chi_{i}\right|^{2}\right)^{p /(p-1)} w^{n /(2 s(p-1))} d x\right\}^{2 s(p-1) / n} \\
& +2^{4 s p / n-1}\left\{\int_{\mathbb{R}^{n}}\left(\sum_{i=1}^{N}\left|\chi_{i}\right|^{2}\right)^{p /(p-1)} w^{n /(2 s(p-1))} d x\right\}^{2 s(p-1) / n} \\
& \leq c 2^{4 s p / n-1} N^{2 s p / n-1} \varepsilon^{2 s p / n} \\
& +c 2^{6 s p / n-2}(K+\varepsilon)^{2 s p / n-1} \sum_{i=1}^{N} \int_{\mathbb{R}^{n}}\left|(-\Delta)^{s / 2} \chi_{i}\right|^{2} w d x,
\end{aligned}
$$

where we used (3.11) and (3.1) for $\chi_{i}$. Since

$$
\begin{aligned}
& \sum_{i=1}^{N} \int_{\mathbb{R}^{n}}\left|(-\Delta)^{s / 2} \chi_{i}\right|^{2} w d x \\
& \quad \leq 2 \sum_{i=1}^{N} \int_{\mathbb{R}^{n}}\left|(-\Delta)^{s / 2} \chi_{i}-(-\Delta)^{s / 2} \phi_{i}\right|^{2} w d x+2 \sum_{i=1}^{N} \int_{\mathbb{R}^{n}}\left|(-\Delta)^{s / 2} \phi_{i}\right|^{2} w d x \\
& \quad \leq 2 \varepsilon+2 \sum_{i=1}^{N} \int_{\mathbb{R}^{n}}\left|(-\Delta)^{s / 2} \phi_{i}\right|^{2} w d x,
\end{aligned}
$$

we have by (3.12)

$$
\begin{aligned}
& \left\{\int_{\mathbb{R}^{n}}\left(\sum_{i=1}^{N}\left|\phi_{i}\right|^{2}\right)^{p /(p-1)} w^{n /(2 s(p-1))} d x\right\}^{2 s(p-1) / n} \\
& \leq c 2^{4 s p / n-1} N^{2 s p / n-1} \varepsilon^{2 s p / n}+c 2^{6 s p / n-1}(K+\varepsilon)^{2 s p / n-1} \varepsilon \\
& \quad+c 2^{6 s p / n-1}(K+\varepsilon)^{2 s p / n-1} \sum_{i=1}^{N} \int_{\mathbb{R}^{n}}\left|(-\Delta)^{s / 2} \phi_{i}\right|^{2} w d x .
\end{aligned}
$$

Since we can take $\varepsilon$ arbitrary small, we conclude

$$
\begin{aligned}
\left\{\int_{\mathbb{R}^{n}}\left(\sum_{i=1}^{N}\left|\phi_{i}\right|^{2}\right)^{p /(p-1)}\right. & \left.w^{n /(2 s(p-1))} d x\right\}^{2 s(p-1) / n} \\
& \leq c 2^{6 s p / n-1} K^{2 s p / n-1} \sum_{i=1}^{N} \int_{\mathbb{R}^{n}}\left|(-\Delta)^{s / 2} \phi_{i}\right|^{2} w d x
\end{aligned}
$$

Hence we get (3.1). 


\section{Proof of Lemma 3.3}

The arguments of the proof are similar to those in [11] and [12]. First we consider the case $n>2 s$. For $\lambda>0$ we set

$$
\mathcal{I}_{\lambda}=\left\{Q \in \mathcal{Q}: \alpha|Q|^{-2 s / n-1} \int_{Q} w d x-\beta|Q|^{-1} \int_{Q} v d x<-\lambda\right\} .
$$

Then we have for $Q \in \mathcal{I}_{\lambda}$

$$
\alpha|Q|^{-2 s / n-1} \int_{Q} w d x<|Q|^{-1} \int_{Q}(\beta v-\lambda)_{+} d x,
$$

where

$$
(\beta v-\lambda)_{+}(x)=\max \{0, \beta v(x)-\lambda\} .
$$

Since $p=n /(2 s)+\gamma, \gamma \in(0,1]$, and

$$
\beta^{-p} \gamma \int_{0}^{\infty} \int_{\beta v>\lambda}(\beta v-\lambda)^{n /(2 s)} w^{-n /(2 s)} d x \lambda^{\gamma-1} d \lambda \leq \int_{\mathbb{R}^{n}} v^{p} w^{-n /(2 s)} d x<\infty,
$$

we have

$$
\int_{\mathbb{R}^{n}}(\beta v-\lambda)_{+}^{n /(2 s)} w^{-n /(2 s)} d x<\infty
$$

for all $\lambda>0$. By the assumption $w^{-n /(2 s)} \in A_{n /(2 s)}$ and (ii) of Proposition 2.1, there exists a $\kappa^{\prime} \in(1, n /(2 s))$ such that $w^{-n /(2 s)} \in A_{n /\left(2 s \kappa^{\prime}\right)}$. We set

$$
v_{\lambda}^{*}(x)=M\left((\beta v-\lambda)_{+}^{\kappa^{\prime}}\right)(x)^{1 / \kappa^{\prime}} .
$$

Then

$$
\int_{\mathbb{R}^{n}}\left(v_{\lambda}^{*}\right)^{n /(2 s)} w^{-n /(2 s)} d x \leq c_{1} \int_{\mathbb{R}^{n}}(\beta v-\lambda)_{+}^{n /(2 s)} w^{-n /(2 s)} d x<\infty
$$

and $v_{\lambda}^{*} \in A_{1}$ by (iii) of Proposition 2.1, where $c_{1}$ depends only on $n, s$ and $A_{n /(2 s)^{-}}$constant of $w^{-n /(2 s)}$.

We can show that $\mathcal{I}_{\lambda}$ is a finite set as follows. Let $Q \in \mathcal{I}_{\lambda}$. Then we have

$$
\begin{aligned}
\alpha|Q|^{-2 s / n} \int_{Q} w d x & \leq \int_{Q} v_{\lambda}^{*} d x \\
& \leq\left\{\int_{Q}\left(v_{\lambda}^{*}\right)^{n /(2 s)} w^{-n /(2 s)} d x\right\}^{2 s / n}\left\{\int_{Q} w^{n /(n-2 s)} d x\right\}^{(n-2 s) / n} .
\end{aligned}
$$


Since $w^{-n /(2 s)} \in A_{n /(2 s)}$, the last quantity is bounded by

$$
\begin{aligned}
c_{2}\left\{\int_{Q}\left(v_{\lambda}^{*}\right)^{n /(2 s)}\right. & \left.w^{-n /(2 s)} d x\right\}^{2 s / n}|Q|\left(\int_{Q} w^{-n /(2 s)} d x\right)^{-2 s / n} \\
& \leq c_{2}\left\{\int_{Q}\left(v_{\lambda}^{*}\right)^{n /(2 s)} w^{-n /(2 s)} d x\right\}^{2 s / n}|Q|^{-2 s / n} \int_{Q} w d x
\end{aligned}
$$

where we used the inequality

$$
1 \leq \frac{1}{|Q|} \int_{Q} w d x\left(\frac{1}{|Q|} \int_{Q} w^{-n /(2 s)} d x\right)^{2 s / n} .
$$

The above calculation says

$$
1 \leq c_{3} \int_{Q}\left(v_{\lambda}^{*}\right)^{n /(2 s)} w^{-n /(2 s)} d x
$$

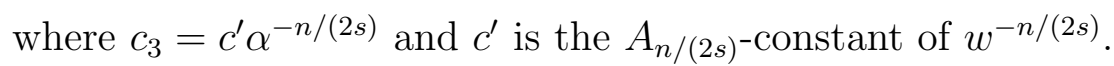

First we assume that $\mathcal{I}_{\lambda}$ includes infinite disjoint cubes $\left\{Q_{i}\right\}_{i=1}^{\infty}$. Then we have

$\infty=\sum_{i=1}^{\infty} 1 \leq \sum_{i=1}^{\infty} c_{3} \int_{Q_{i}}\left(v_{\lambda}^{*}\right)^{n /(2 s)} w^{-n /(2 s)} d x \leq c_{3} \int_{\mathbb{R}^{n}}\left(v_{\lambda}^{*}\right)^{n /(2 s)} w^{-n /(2 s)} d x<\infty$.

This is a contradiction. Hence $\mathcal{I}_{\lambda}$ does not include infinite disjoint cubes.

Next we assume that there exist infinite cubes $\left\{Q_{i}\right\}_{i=1}^{\infty} \subset \mathcal{I}_{\lambda}$ such that $Q_{i} \neq Q_{j}(i \neq j)$ and $Q_{1} \subset Q_{2} \subset Q_{3} \subset \cdots$. Let $\tilde{Q}_{i}$ be a half size dyadic sub-cube of $Q_{i+1}$ such that $Q_{i} \cap \tilde{Q}_{i}=\emptyset$. Since $Q_{i+1} \in \mathcal{I}_{\lambda}$, we have

$$
\alpha\left|Q_{i+1}\right|^{-2 s / n} \int_{Q_{i+1}} w d x \leq \int_{Q_{i+1}} v_{\lambda}^{*} d x .
$$

Now we get

$$
\int_{Q_{i+1}} v_{\lambda}^{*} d x \leq \int_{3 \tilde{Q}_{i}} v_{\lambda}^{*} d x \leq c_{4} \int_{\tilde{Q}_{i}} v_{\lambda}^{*} d x
$$

where we used the doubling property of $v_{\lambda}^{*}$. Since

$$
\left|Q_{i+1}\right|^{-2 s / n} \int_{Q_{i+1}} w d x \geq 2^{-2 s}\left|\tilde{Q}_{i}\right|^{-2 s / n} \int_{\tilde{Q}_{i}} w d x
$$

we get

$$
c_{5}\left|\tilde{Q}_{i}\right|^{-2 s / n} \int_{\tilde{Q}_{i}} w d x \leq \int_{\tilde{Q}_{i}} v_{\lambda}^{*} d x
$$


The similar calculation as before leads to

$$
1 \leq c_{6} \int_{\tilde{Q}_{i}}\left(v_{\lambda}^{*}\right)^{n /(2 s)} w^{-n /(2 s)} d x
$$

where $c_{6}=c^{\prime \prime} \alpha^{-n /(2 s)}$ and $c^{\prime \prime}$ depends only on $n, s$, and $w$. Since $\left\{\tilde{Q}_{i}\right\}_{i=1}^{\infty}$ is a set of infinite disjoint cubes, we have a contradiction as before. Hence any sequence in $\mathcal{I}_{\lambda}$ such that $Q_{1} \subset Q_{2} \subset Q_{3} \subset \cdots$ has a maximal element. Similarly we can show that any sequence in $\mathcal{I}_{\lambda}$ such that $Q_{1} \supset Q_{2} \supset Q_{3} \supset \cdots$ has a minimal element.

By these arguments the number of maximal cubes and minimal cubes in $\mathcal{I}_{\lambda}$ with respect to the inclusion relation is finite. Hence $\mathcal{I}_{\lambda}$ is a finite set. We remark that the non-decreasing rearrangement of $\mathcal{I}$ in (3.6) is possible because $\mathcal{I}_{\lambda}$ is a finite set for every $\lambda>0$.

Let $N(\lambda)=\sharp \mathcal{I}_{\lambda}$, that is, the number of elements of $\mathcal{I}_{\lambda}$. Let $\tilde{\mathcal{I}}_{\lambda}$ be the set of all $Q \in \mathcal{I}_{\lambda}$ which satisfy the following condition: there exists a half size dyadic sub-cube $\tilde{Q} \subset Q$ such that $\tilde{Q} \notin \mathcal{I}_{\lambda}$ and $\tilde{Q}$ does not contain any dyadic cube in $\mathcal{I}_{\lambda}$. Then we have the following lemma.

Lemma 4.1. $\sharp \mathcal{I}_{\lambda} \leq 2 \sharp \tilde{\mathcal{I}}_{\lambda}$.

Lemma 4.1 is proved in Rochberg and Taibleson's paper ([9, Lemma 1]).

Let $Q \in \tilde{\mathcal{I}}_{\lambda}$ and $\tilde{Q}$ be a dyadic cube which satisfies the condition in the definition of $\tilde{\mathcal{I}}_{\lambda}$. Then by similar calculations as before we get

$$
1 \leq c_{6} \int_{\tilde{Q}}\left(v_{\lambda}^{*}\right)^{n /(2 s)} w^{-n /(2 s)} d x
$$

For every $Q \in \tilde{\mathcal{I}}_{\lambda}$ we choose a $\tilde{Q}$ as above. Let $\left\{\tilde{Q}_{j}\right\}_{j \in J}$ be the set of all such cubes $\tilde{Q}$. Then the cubes in $\left\{\tilde{Q}_{j}\right\}_{j \in J}$ are mutually disjoint. Therefore we get

$$
\begin{aligned}
\sharp \tilde{\mathcal{I}}_{\lambda}=\sharp J & \leq \sum_{j \in J} c_{6} \int_{\tilde{Q}_{j}}\left(v_{\lambda}^{*}\right)^{n /(2 s)} w^{-n /(2 s)} d x \\
& \leq c_{6} \int_{\mathbb{R}^{n}}\left(v_{\lambda}^{*}\right)^{n /(2 s)} w^{-n /(2 s)} d x \leq c_{7} \int_{\mathbb{R}^{n}}(\beta v-\lambda)_{+}^{n /(2 s)} w^{-n /(2 s)} d x,
\end{aligned}
$$

where we used (4.2). Hence we have

$$
N(\lambda) \leq 2 c_{7} \int_{\mathbb{R}^{n}}(\beta v-\lambda)_{+}^{n /(2 s)} w^{-n /(2 s)} d x
$$


Therefore we conclude

$$
\begin{aligned}
\sum_{k}\left|\mu_{k}\right|^{\gamma} & =\int_{0}^{\infty} \gamma \lambda^{\gamma-1} N(\lambda) d \lambda \\
& \leq 2 c_{7} \int_{0}^{\infty} \int_{\beta v>\lambda}(\beta v-\lambda)_{+}^{n /(2 s)} w^{-n /(2 s)} d x \gamma \lambda^{\gamma-1} d \lambda \\
& \leq c_{8} \int_{\mathbb{R}^{n}} v^{p} w^{-n /(2 s)} d x
\end{aligned}
$$

where $c_{8}=c^{\prime \prime \prime} \alpha^{-n /(2 s)} \beta^{p}$ and $c^{\prime \prime \prime}$ depends only on $n, s, p$ and $w$.

Next we consider the case $n \leq 2 s$. We remark that $v(x)>0$ for all $x \in \mathbb{R}^{n}$. In fact if $v\left(x_{0}\right)=0$ at some point $x_{0}$, then by the definition of the maximal operator we have $V \equiv 0$, that is, $\phi_{i} \equiv 0, i=1, \ldots, N$.

We also remark that $\mathcal{I}$ in (3.6) is not empty. In fact if $\mathcal{I}$ is empty, then we have

$$
\beta \int_{Q} v d x \leq \alpha|Q|^{-2 s / n} \int_{Q} w d x
$$

for all $Q \in \mathcal{Q}$. Let $Q_{0} \in \mathcal{Q}$ and $Q_{0} \subset Q_{1} \subset Q_{2} \subset \cdots$ be the infinite sequence of dyadic cubes such that $Q_{i+1}$ is the parent of $Q_{i}$ for all $i=0,1,2, \ldots$. By (1.2) we have

$$
\left|Q_{i+1}\right|^{-2 s / n} \int_{Q_{i+1}} w d x \leq\left|Q_{i}\right|^{-2 s / n} \int_{Q_{i}} w d x \quad \text { for all } i .
$$

Hence we have

$$
\beta \int_{Q_{i}} v d x \leq \alpha\left|Q_{0}\right|^{-2 s / n} \int_{Q_{0}} w d x
$$

for all $i$. On the other hand, since $v \in A_{1}$, there exists a constant $d>1$ such that

$$
d \int_{Q_{i}} v d x \leq \int_{Q_{i+1}} v d x
$$

for all $i$ (c.f. [5, p. 141]). Hence we have

$$
d^{i} \int_{Q_{0}} v d x \leq \int_{Q_{i}} v d x
$$

and

$$
\lim _{i \rightarrow \infty} \int_{Q_{i}} v d x=\infty,
$$

which contradicts to (4.3). Therefore $\mathcal{I}$ is not empty. 
Let $Q \in \mathcal{I}$ and $Q^{\prime}$ be the parent of $Q$. Then we have

$$
\alpha\left|Q^{\prime}\right|^{-2 s / n} \int_{Q^{\prime}} w d x \leq \alpha|Q|^{-2 s / n} \int_{Q} w d x<\beta \int_{Q} v d x \leq \beta \int_{Q^{\prime}} v d x,
$$

where we used the assumption (1.2). Hence we have $Q^{\prime} \in \mathcal{I}$, which means that $\mathcal{I}$ is an infinite set.

Lemma 4.2. There exists a $c>0$ such that

$$
\sum_{Q \in \mathcal{I}}\left(\frac{1}{|Q|} \int_{Q} v d x\right)^{\gamma} \leq c \int_{\mathbb{R}^{n}} v^{p} w^{-n /(2 s)} d x,
$$

where $c=c^{\prime} \alpha^{-n /(2 s)} \beta^{n /(2 s)}$ and $c^{\prime}$ depends only on $n, p, s$ and $w$.

This lemma is proved in [12, Lemma 3.3]. Let $\mathcal{I}_{\lambda}$ be the set defined by (4.1).

Lemma 4.3. For each $\lambda>0, \mathcal{I}_{\lambda}$ is a finite set.

Lemma 4.3 is easily proved by Lemma 4.2 (cf. [12, Lemma 3.4]). By Lemma 4.3 we can show that the non-decreasing rearrangement of $\mathcal{I}$ is possible.

By Lemma 4.2 we conclude

$$
\begin{aligned}
\sum_{k=1}^{\infty}\left|\mu_{k}\right|^{\gamma} & =c \sum_{Q \in \mathcal{I}}\left(\beta|Q|^{-1} \int_{Q} v d x-\alpha|Q|^{-2 s / n-1} \int_{Q} w d x\right)^{\gamma} \\
& \leq c \sum_{Q \in \mathcal{I}}\left(\beta|Q|^{-1} \int_{Q} v d x\right)^{\gamma} \leq c \int_{\mathbb{R}^{n}} v^{p} w^{-n /(2 s)} d x
\end{aligned}
$$

where $c=c^{\prime \prime} \alpha^{-n /(2 s)} \beta^{p}$ and $c^{\prime \prime}$ depends only on $n, p, s$ and $w$. This ends the proof of Lemma 3.3.

\section{Proof of Lemmas 3.1 and 3.2}

In this section we give a proof of Lemmas 3.1 and 3.2. The following argument is in [11]. We use the following lemma.

Lemma 5.1. Let $w \in A_{2}$ and $m \in C^{n}\left(\mathbb{R}^{n} \backslash\{0\}\right)$. Suppose that

$$
B=\max _{|\sigma| \leq n} \sup _{0<r} r^{2|\sigma|-n} \int_{r \leq|\xi| \leq 2 r}\left|\left(\frac{\partial}{\partial \xi}\right)^{\sigma} m(\xi)\right|^{2} d \xi<\infty .
$$

Then the operator $T$ defined by $\widehat{T f}(\xi)=m(\xi) \hat{f}(\xi)$ is bounded from $L^{2}(w)$ to $L^{2}(w)$ and the operator norm $\|T\|$ is bounded by $C B^{1 / 2}$ where $C$ is a constant which depends only on $n$ and $w$.

The proof of Lemma 5.1 is in [6] or [7]. 
For $\nu \in \mathbb{Z}$ we define $\psi_{\nu}(x)=2^{n \nu} \psi\left(2^{\nu} x\right)$. Let $w \in A_{2}$ and $s \geq 0$. Frazier and Jawerth proved that there exist positive constants $c_{1}$ and $c_{2}$ such that

$$
\begin{aligned}
c_{1} \sum_{Q \in \mathcal{Q}}|Q|^{-2 s / n}\left|\left(f, \psi_{Q}\right)\right|^{2} \frac{1}{|Q|} \int_{Q} w d x & \leq \int_{\mathbb{R}^{n}}\left\{\sum_{\nu \in \mathbb{Z}} 2^{2 s \nu}\left|f * \psi_{\nu}(x)\right|^{2}\right\} w(x) d x \\
& \leq c_{2} \sum_{Q \in \mathcal{Q}}|Q|^{-2 s / n}\left|\left(f, \psi_{Q}\right)\right|^{2} \frac{1}{|Q|} \int_{Q} w d x
\end{aligned}
$$

for all $f \in C_{0}^{\infty}\left(\mathbb{R}^{n}\right)$ where $c_{1}$ and $c_{2}$ depend only on $n, s$ and $w$ ([3, Proposition 10.14]).

We shall use the argument in Kurtz [6, p.242, p.243]. Let $\left\{r_{\nu}(t)\right\}$ be the Rademacher functions on $[0,1]$ indexed by $\nu \in \mathbb{Z}$ and

$$
T_{t} f(x)=\sum_{\nu \in \mathbb{Z}} r_{\nu}(t) f * \psi_{\nu}(x)
$$

Then $T_{t}$ satisfies the condition of Lemma 5.1. Hence

$$
\int_{\mathbb{R}^{n}}\left|T_{t} f(x)\right|^{2} w(x) d x \leq C M \int_{\mathbb{R}^{n}}|f(x)|^{2} w(x) d x,
$$

for all $f \in C_{0}^{\infty}\left(\mathbb{R}^{n}\right)$ where

$$
M=\max _{|\sigma| \leq n}\left\|\partial^{\sigma} \hat{\psi}\right\|_{\infty}^{2}
$$

and $C$ is a positive constant depending only on $n$ and $w$. By integrating from 0 to 1 with respect to $t$, we get

$$
\int_{\mathbb{R}^{n}}\left\{\sum_{\nu \in \mathbb{Z}}\left|f * \psi_{\nu}(x)\right|^{2}\right\} w(x) d x \leq C M \int_{\mathbb{R}^{n}}|f(x)|^{2} w(x) d x .
$$

By the duality argument and the fact $w^{-1} \in A_{2}$ we obtain

$$
\int_{\mathbb{R}^{n}}|f(x)|^{2} w(x) d x \leq C M \int_{\mathbb{R}^{n}}\left\{\sum_{\nu \in \mathbb{Z}}\left|f * \psi_{\nu}(x)\right|^{2}\right\} w(x) d x
$$

for all $f \in C_{0}^{\infty}\left(\mathbb{R}^{n}\right)$. Hence we have

$$
c_{3} M^{-1} \int_{\mathbb{R}^{n}}|f|^{2} w d x \leq \int_{\mathbb{R}^{n}}\left\{\sum_{\nu \in \mathbb{Z}}\left|f * \psi_{\nu}\right|^{2}\right\} w d x \leq c_{4} M \int_{\mathbb{R}^{n}}|f|^{2} w d x,
$$

where $c_{3}$ and $c_{4}$ are constants depending only on $n$ and $w$. 
Therefore we get

$$
\begin{aligned}
c_{3} M^{-1} \int_{\mathbb{R}^{n}}\left|(-\Delta)^{s / 2} f\right|^{2} w d x & \leq \int_{\mathbb{R}^{n}}\left\{\sum_{\nu \in \mathbb{Z}}\left|(-\Delta)^{s / 2} f * \psi_{\nu}\right|^{2}\right\} w d x \\
& \leq c_{4} M \int_{\mathbb{R}^{n}}\left|(-\Delta)^{s / 2} f\right|^{2} w d x
\end{aligned}
$$

for all $f \in C_{0}^{\infty}\left(\mathbb{R}^{n}\right)$ (c.f.[11]).

Let $\Phi \in \mathcal{S}\left(\mathbb{R}^{n}\right)$ satisfy $\operatorname{supp} \Phi \subset\{\xi: 1 / 4 \leq|\xi| \leq 4\}$ and $\Phi(\xi)=1$ for $1 / 2 \leq|\xi| \leq 2$. For $\nu \in \mathbb{Z}$ the multiplier $m_{\nu}(\xi)=2^{-s \nu}|\xi|{ }^{s} \Phi\left(\xi / 2^{\nu}\right)$ satisfies the condition of Lemma 5.1. Hence we have

$$
\int_{\mathbb{R}^{n}}\left|(-\Delta)^{s / 2} f * \psi_{\nu}(x)\right|^{2} w(x) d x \leq c_{5} \int_{\mathbb{R}^{n}} 2^{2 s \nu}\left|f * \psi_{\nu}(x)\right|^{2} w(x) d x,
$$

where

$$
c_{5}=c_{6} \inf _{\Phi} \max _{|\sigma| \leq n}\left\|\partial^{\sigma} \Phi\right\|_{\infty}^{2}
$$

and $c_{6}$ is a positive constant depending only on $n, s$ and $w$ and the infimum is taken over all possible $\Phi$.

Similarly there exists a positive constant $c_{7}$ depending only on $n, s$ and $w$ such that

$$
\int_{\mathbb{R}^{n}} 2^{2 s \nu}\left|f * \psi_{\nu}(x)\right|^{2} w(x) d x \leq c_{7} \int_{\mathbb{R}^{n}}\left|(-\Delta)^{s / 2} f * \psi_{\nu}(x)\right|^{2} w(x) d x .
$$

Hence we get

$$
\begin{aligned}
c_{8} M^{-1} \int_{\mathbb{R}^{n}}\left|(-\Delta)^{s / 2} f\right|^{2} w d x & \leq \sum_{Q \in \mathcal{Q}}|Q|^{-2 s / n}\left|\left(f, \psi_{Q}\right)\right|^{2} \frac{1}{|Q|} \int_{Q} w d x \\
& \leq c_{9} M \int_{\mathbb{R}^{n}}\left|(-\Delta)^{s / 2} f\right|^{2} w d x
\end{aligned}
$$

for all $f \in C_{0}^{\infty}\left(\mathbb{R}^{n}\right)$, where $c_{8}$ and $c_{9}$ are positive constant depending only on $n, s$ and $w$. This ends the proof of Lemmas 3.1 and 3.2.

\section{References}

[1] Daubechies, I.: Ten lectures on wavelets. CBMS-NSF Regional Conference Series in Applied Mathematics 61. SIAM, 1992.

[2] Edmunds, D. E. And Ilyin, A. A.: On some multiplicative inequalities and approximation numbers. Quart. J. Math. Oxford Ser. (2) 45 (1994) 159-179. 
[3] Frazier, M. and Jawerth, B.: A discrete transform and decompositions of distribution spaces. J. Funct. Anal. 93 (1990), 34-170.

[4] Ghidaglia, J.-M., Marion, M. and Temam, R.: Generalization of the Sobolev-Lieb-Thirring inequalities and applications to the dimension of attractors. Differential Integral Equations 1 (1988), 1-21.

[5] García-Cuerva, J. and Rubio de Francia, J. L.: Weighted norm inequalities and related topics. North-Holland Mathematics Studies 116. North-Holland Publishing Co., Amsterdam, 1985.

[6] Kurtz, D. S.: Littlewood-Paley and multiplier theorems on weighted $L^{p}$ spaces. Trans. Amer. Math. Soc. 259 (1980), 235-254.

[7] Kurtz, D. S. and Wheeden, R. L.: Results on weighted norm inequalities for multipliers. Trans. Amer. Math. Soc. 255 (1979), 343-362.

[8] Lieb, E. AND ThiRring, W.: Inequalities for the moments of the eigenvalues of the Schrödinger hamiltonian and their relation to Sobolev inequalities. In Studies in Mathematical Physics, 269-303. Princeton University Press, 1976.

[9] Rochberg, R. and Taibleson, M.: An averaging operator on a tree. In Harmonic analysis and partial differential equations (El Escorial), 207-213. Lecture Notes in Math. 1384. Springer-Verlag, 1989.

[10] Stein, E. M.: Harmonic analysis: real-variable methods, orthogonality, and oscillatory integrals. Princeton Mathematical Series 43. Monographs in Harmonic Analysis, III. Princeton University Press, 1993.

[11] Tachizawa, K.: On the moments of negative eigenvalues of elliptic operators. J. Fourier Anal. Appl. 8 (2002), 233-244.

[12] TACHIzAwA, K.: A generalization of the Lieb-Thirring inequalities in low dimensions. Hokkaido Math. J. 32 (2003), 383-399.

[13] Temam, R.: Infinite-dimensional dynamical systems in mechanics and physics. Applied Mathematical Sciences 68. Springer, New York, 1988.

Recibido: 25 de octubre de 2002

Revisado: 22 de septiembre de 2003

Kazuya Tachizawa

Department of Mathematics

Faculty of Science, Hokkaido University

Sapporo 060-0810

Japan

tachizaw@math.sci.hokudai.ac.jp

The author was partly supported by the Grants-in-Aid for Scientific Research, The Ministry of Education, Science, Sports and Culture of Japan. 